\title{
Vascular Malformations: A Review
}

\author{
Joshua A. Cox, MD ${ }^{1}$ Erica Bartlett, MD ${ }^{1}$ Edward I. Lee, MD ${ }^{1}$ \\ ${ }^{1}$ Division of Plastic Surgery, Baylor College of Medicine, \\ Houston, Texas \\ Address for correspondence Edward I. Lee, MD, Division of Plastic \\ Surgery, Baylor College of Medicine, 6701 Fannin Street, Suite 610, \\ Houston, TX 77030 (e-mail: eilee@bcm.edu).
} Semin Plast Surg 2014;28:58-63.

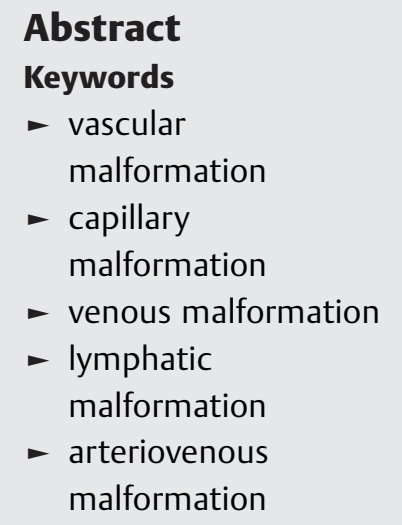

Identification and treatment of vascular malformations is a challenging endeavor for physicians, especially given the great concern and anxiety created for patients and their families. The goal of this article is to provide a review of vascular malformations, organized by subtype, including capillary, venous, lymphatic and arteriovenous malformations. Only by developing a clear understanding of the clinical aspects, diagnostic tools, imaging modalities, and options for intervention will appropriate care be provided and results maximized.
Vascular anomalies, a group encompassing a wide variety of lesions related to the disorder of vascular development, remain both diagnostic and treatment challenges to treating physicians. The terminology used to describe and classify vascular anomalies is the key for proper diagnosis and treatment. The classification system established by the International Society for the Study of Vascular Anomalies (ISSVA) is now a widely accepted system used to categorize vascular anomalies into two types: (1) vasoproliferative or vascular neoplasms such as hemangioma, and (2) vascular malformations. ${ }^{1}$

The distinction between the two is based on histopathological assessment of increased cell turnover. Vascular tumors, formerly classified as hemangiomas, are true neoplasms with pathologic cell proliferation. These tumors typically exhibit rapid postnatal growth and slow regression into late childhood. ${ }^{2}$ Vascular malformations, on the other hand, are comprised of abnormally formed channels within a vascular apparatus that are lined by endothelial cells and do not undergo abnormal cellular turnover. They too are congenital in nature, but often go unnoticed at birth, never regress, and grow proportionally with the individual. ${ }^{3}$

In this article, we will focus solely on vascular malformations, reviewing the basic nomenclature, etiology, and diagnostic criteria for each subcategory and discussing treatment options available today. An in-depth review of vascular malformations associated with known syndromes as well as vascular tumors will be provided in separate articles. It is important to be able to make accurate diagnosis, understand the basic physiology, and use appropriate diagnostic and treatment modalities to optimize outcome.

\section{Vascular Malformations}

Vascular malformations are grouped together based upon their common embryological origin of having a single endothelial cell lining. ${ }^{3}$ Vascular malformations are thought to result from developmental errors during embryogenesis, such as abnormal signaling processes that control apoptosis, maturation, and growth of vascular cells. These errors lead to the persistence of vascular plexus cells with a certain degree of differentiation. ${ }^{4}$ There are four major categories of vascular malformations based on their flow characteristics: slow-flow (capillary malformation, venous malformation, lymphatic malformation) and fast-flow (arteriovenous malformation). These lesions often have components of multiple malformations, such as a mixed lymphatico-venous malformation, further adding to the confusion with respect to proper nomenclature (-Fig. 1).

As with any medical or surgical anomaly, accurate diagnosis is paramount to successful treatment. With vascular
Issue Theme Vascular Anomalies; Guest Editor, Edward I. Lee, MD
Copyright @ 2014 by Thieme Medical Publishers, Inc., 333 Seventh Avenue, New York, NY 10001, USA. Tel: +1(212) 584-4662.
DOI http://dx.doi.org/ 10.1055/s-0034-1376263. ISSN 1535-2188. 


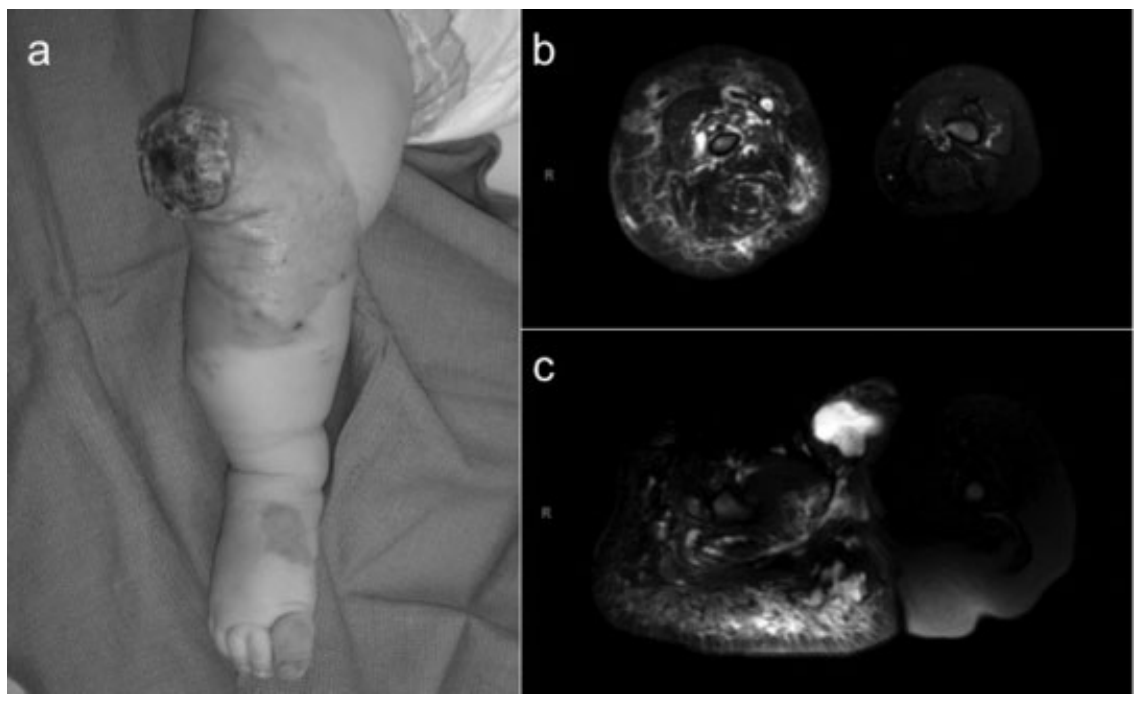

Fig. 1 Mixed lymphatico-venous malformation (LVM) involving lower extremity. (A) Ulceration is an indication for intervention. (B) Axial, contrastenhanced magnetic resonance image shows both irregular but extensive, contrast-enhancing lesion indicative of a venous malformation of the lateral thigh as well as (C) cystic, nonenhancing densities along gluteus muscle and pararectal area indicative of a lymphatic malformation.

malformations, a thorough history and physical exam will allow the astute physician to make a sound diagnosis for a majority of the clinical cases presented. Additionally, proper imaging modalities such as ultrasound (US) with gray scale, color Doppler and spectral Doppler tracings, or magnetic resonance imaging (MRI) can aid when the diagnosis is in question, as there may be overlap in clinical appearance in these anomalies. ${ }^{5}$ These imaging modalities can assist in confirming particular attributes of the lesion, defining anatomic locations/boundaries, and planning potential surgical intervention.

In general, the management of vascular malformation is expectant in nature with both noninvasive and invasive treatment of symptomatic lesions. Lesions located in the head and neck region, however, require special attention as they can cause obstruction of critical structures such as the visual axis or the airway (-Fig. 2).

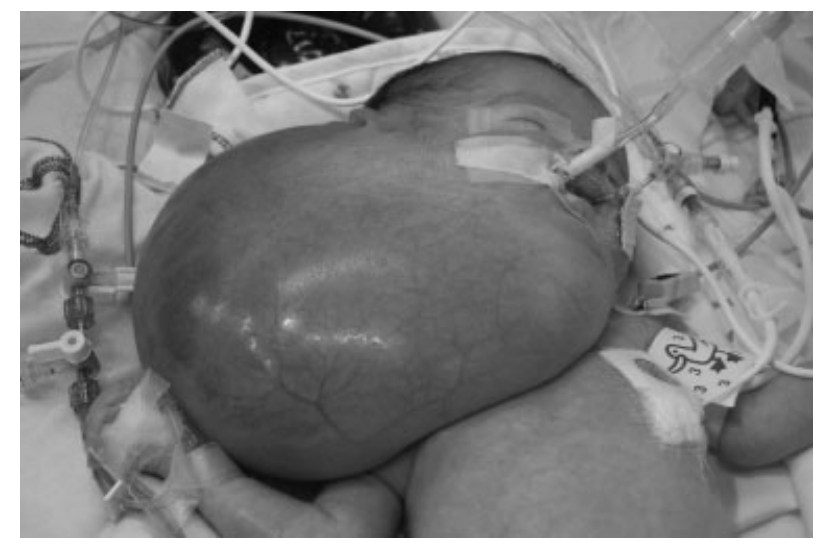

Fig. 2 Very large lymphatic malformation involving the right head and neck region and compressing the airway.

\section{Capillary Malformation}

Capillary malformations (CMs) affect the capillaries in the papillary dermis and commonly appear as a macular, pink or purple stain that is present at birth and persists throughout life. ${ }^{6}$ Capillary malformations, found in $0.5 \%$ of the population, were initially referred to as "port-wine stain," which is inaccurate, but has persisted due to its widespread use throughout the literature. The majority of CMs appear in the face and tend to be in the trigeminal nerve distribution, especially ophthalmic (V1) and maxillary (V2) divisions. ${ }^{7}$ Capillary malformations in the V1 or midline distribution should alert physicians of possible central nervous system (CNS) involvement and warrant imaging studies as this is highly associated with leptomeningeal involvement and subsequent seizure disorders (i.e,, Sturge-Weber syndrome).

As the patient progresses into adulthood, the stains tend to darken and thicken into a "cobblestone" appearance and can distort facial features, including the underlying bony structures. There is currently no imaging modality needed to assist in diagnosis, but MRI is strongly recommended to rule out CNS involvement. Positive findings include gyral enhancement, enlargement and enhancement of ipsilateral choroid plexus, progressive cortical atrophy and calcification. ${ }^{8}$ Cerebral angiography can detect parenchymal contrast stasis and abnormal cortical veins associated with CNS involvement.

The majority of treatment modalities are ablative in nature, such as the pulsed-dye laser (580-595 nm). ${ }^{9}$ Significant improvement in the color of the stain can be seen when laser treatment is begun in infancy and when applied to CMs of the lateral face. Noticeable lightening is typically seen in greater than $75 \%$ of patients. Better results are achieved when therapy is initiated early, thus it is recommended that treatment commence before 6 months of age. Surgery is reserved for lesions that are refractory to ablative treatment or are causing significant disfigurement (-Fig. 3). ${ }^{10}$ 


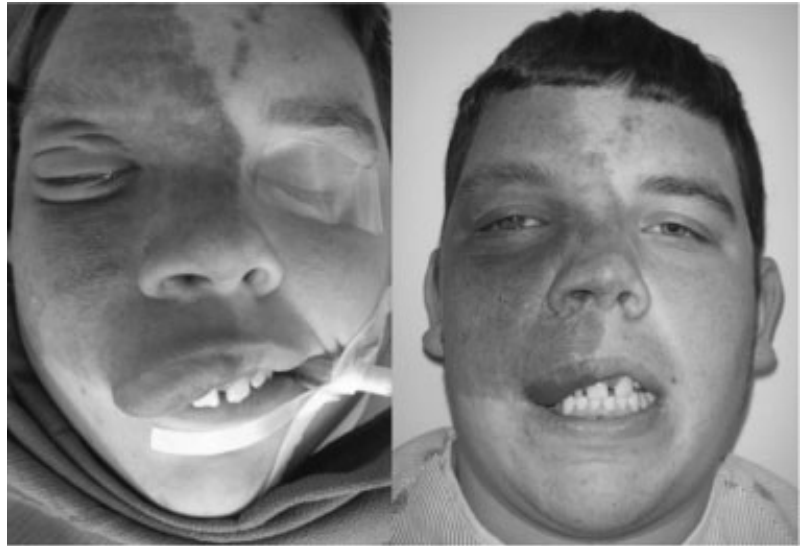

Fig. 3 A 19-year-old man with a capillary malformation in V1 and V2 distribution, status post-serial laser treatments, lip debulking, and excision of redundant tissue through alar and upper lip incision.

\section{Venous Malformations}

Venous malformations (VMs), like other vascular malformations, are present at birth. They are the most common type of vascular malformation, affecting $1 \%$ to $4 \%$ of individuals, and clinically appear as a bluish, soft, compressible lesions typically found on the face, limbs, or trunk ( - Fig. 4). Venous malformations are composed of masses of veins and venulae of different dimensions lined by a single endothelial layer. ${ }^{6}$ Venous malformations are dependent lesions, meaning that they expand and contract based on patient positioning. They tend to grow proportionally with the child and often increase in size with puberty, hormonal changes, or infection. ${ }^{11}$ They show a predisposition to thrombosis, forming phleboliths, which are pathognomonic of VM and are diagnostic on imaging studies. Phleboliths are intralesional calcifications formed as a result of venous stasis and inflammation. ${ }^{12}$

Although US and color Doppler are first-line modalities for diagnosis, showing low flow lesions with phleboliths, MRI is

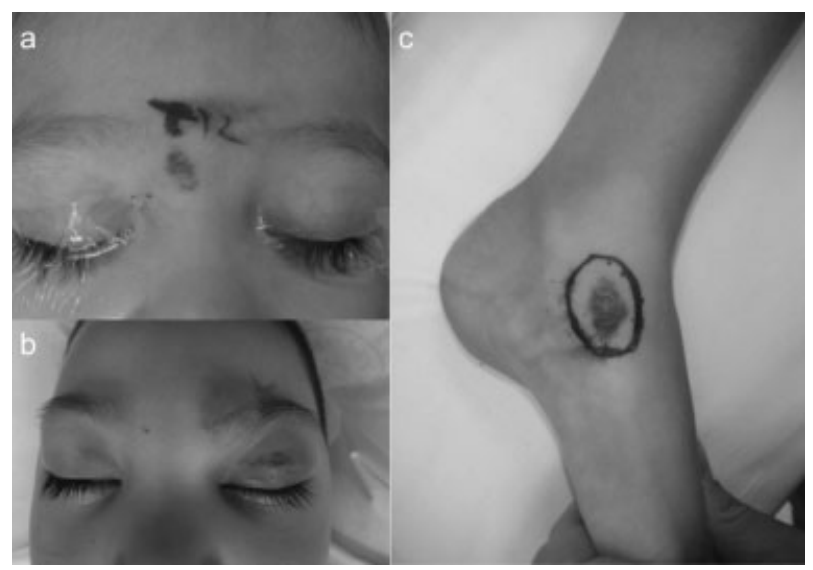

Fig. 4 Various manifestations of localized venous malformations. Venous malformations are bluish, soft, compressible lesions typically found on the $(A, B)$ face, $(C)$ limbs, or trunk. most useful for defining the extent of the disease. ${ }^{13}$ Angiography is also a useful modality for defining extent of disease, especially when identifying deep or small VMs, such as intracranial sinus pericranii or gastrointestinal VM, as traditional MRI and magnetic resonance angiography (MRA) is not as sensitive in identifying these lesions. ${ }^{14}$ Beyond imaging, it is sometimes prudent to obtain a coagulation profile if the patient has a large VM given the risk for localized intravascular coagulopathy. ${ }^{15}$

Treatment options for VM depend on both the extent of the lesion and the location. Typically, functional or aesthetic limitations will drive the initiation of therapy. Interventional radiology can deliver primary treatment such as staged sclerotherapy and embolization, or play a supportive role with pre- or intraoperative embolization. Sclerotherapy with absolute ethanol is effective for treatment of large, extensive VMs, but should be used with caution as it can damage nerves, cause skin necrosis, and induce systemic toxicity. ${ }^{16,17}$ Other common sclerosants used include 3\% sodium tetradecyl sulfate (STS) and bleomycin. Surgery is rarely first-line therapy, but may be considered in select situations, such as (1) to ligate efferent veins to improve the results with sclerotherapy, (2) to remove residual VM after sclerotherapy, (3) to remove lesion resistant to sclerotherapy, or (4) localized lesion amenable to complete excision ( - Fig. 5) ${ }^{18}$ It is important to recognize that resection can be arduous and technically demanding; thus, it should not be undertaken without a thorough discussion of all operative risks.

\section{Lymphatic Malformation}

Lymphatic malformations (LMs) are vascular channels, pouches, or vesicles filled with lymphatic fluid with a single endothelial cell lining, $75 \%$ of which occur in the cervicofacial region (-Fig. 6). They were referred to as "lymphangioma" in the past, which is a misnomer because LMs lack cellular hyperplasia. Lymphatic malformations are categorized by the size of the lymphatic chamber: macrocystic $(>2 \mathrm{~cm})$, microcystic $(<2 \mathrm{~cm})$, or mixed. Lymphatic malformations never regress yet expand and contract based on the amount of lymphatic fluid present and the presence of bleeding or inflammation. ${ }^{6}$ These lesions are often evident at birth and appear as small, crimson dome-shaped nodules as a result of intralesional bleeding. Many macrocystic LMs can enlarge significantly leading to distortion of anatomy, especially of the soft tissues and bones of the face. Along with tissue distortion, frequent bouts of bleeding and cellulitis will dictate the need for intervention.

Most cases of LM are clinically obvious and require no imaging for diagnosis. When a diagnosis is not so clear, imaging modalities such as US and color flow Doppler are particularly useful in establishing a diagnosis. ${ }^{5}$ Ultrasound is performed to identify the characteristic cystic appearance of these lesions. Magnetic resonance imaging is helpful not only in diagnosis, but also for evaluating the extent of the disease. Classic appearance on MRI is mild enhancement of the septae and walls, which creates a characteristic enhancement pattern of rings and arcs. Magnetic resonance imaging is also 


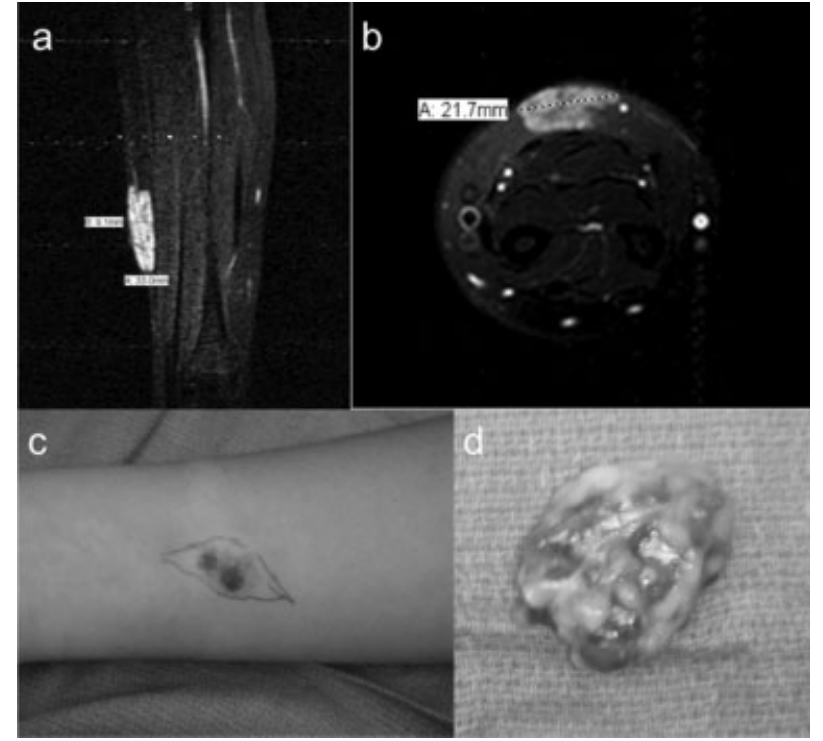

Fig. 5 Venous malformation involving a volar forearm. (A,B) Magnetic resonance imaging (coronal and axial views) shows a contrast-enhancing lesion. (C) Clinically, the mass was bluish, soft, and compressible. (D) Pathologic examination confirmed this mass to be a venous malformation.

useful in distinguishing microcystic versus macrocystic lesions as therapeutic modalities differ between the two. ${ }^{14}$

Treatment options for LM begin with expectant management of symptomatic lesions, such as pain control and

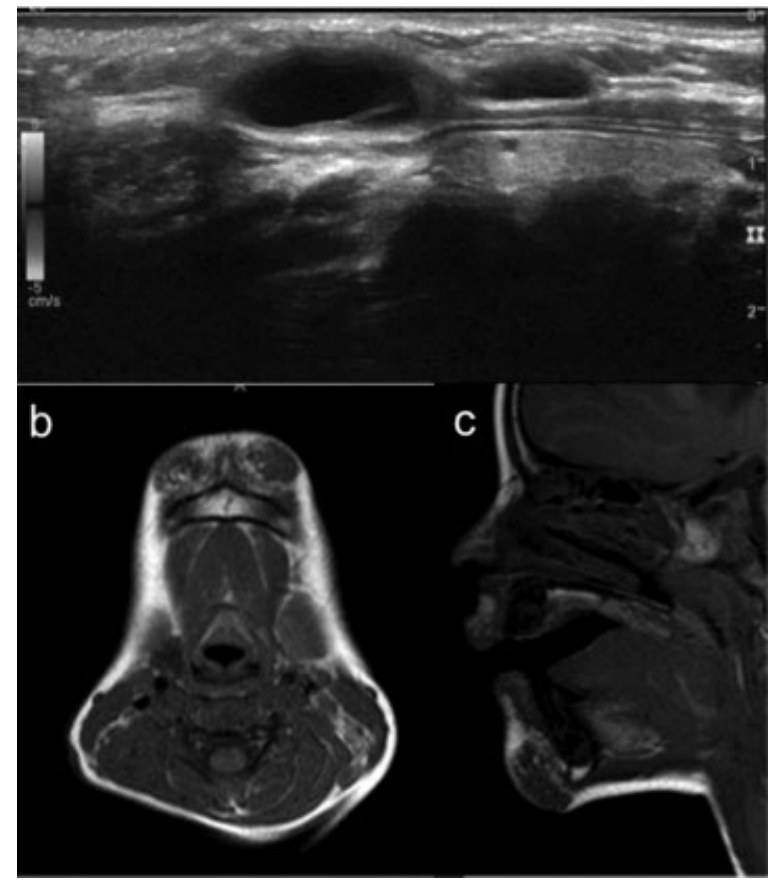

Fig. 6 A 4-year-old boy with lymphatic malformations (LMs) involving the chin, tongue, and sublingual spaces. (A) Ultrasound shows avascular, cystic soft tissue masses consistent with an LM. (B) Axial and (C) sagittal views of contrast-enhanced magnetic resonance imaging show a nonenhancing bilobed chin mass as well as a multispacial lesion involving the tongue and sublingual spaces.

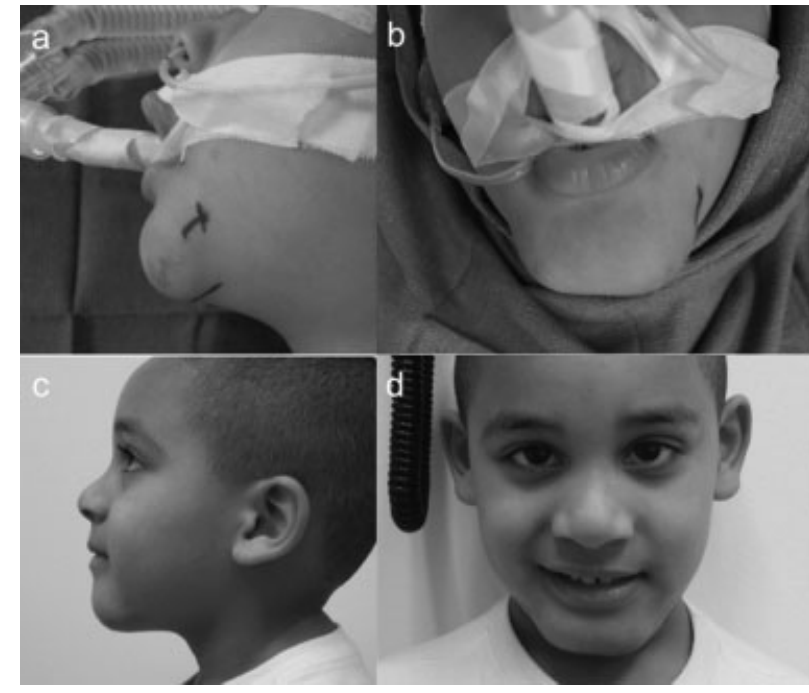

Fig. 7 A 4-year-old boy with lymphatic malformation involving the $(\mathrm{A}, \mathrm{B})$ chin, $(\mathrm{C}, \mathrm{D})$ status postradical resection.

compression for intralesional bleeding and antibiotics for infection, which can often be life threatening. Sclerosants are first-line therapy with options such as absolute ethanol, doxycycline, STS, or picibanil (OK-432). ${ }^{19}$ These agents cause irreversible damage to the endothelium, inducing local inflammation and ultimately fibrosis. The only potentially curative modality is surgical resection. The goals of resection focus on gross debulking of defined anatomic field, limiting blood loss, and minimizing damage to surrounding structures (-Fig. 7). It is important to remember that complete resection is typically not possible as remaining channels will regenerate and extensive radical resection is typically performed at the expense of surrounding normal structures. ${ }^{20,21}$

\section{Arteriovenous Malformation}

Arteriovenous malformations (AVM) represent a class of vascular malformations that develop from an identifiable source vessel called the "nidus," which conducts an abnormal connection of arterial and venous systems. ${ }^{22}$ This type of shunt is usually present at birth, but does not become apparent until the first or second decade of life. Arteriovenous malformations may be slightly compressible and pulsatile with a palpable thrill. This type of lesion is most commonly found intracranially and can expand in response to certain stimuli such as trauma or puberty. Clinically, AVMs can appear in soft tissues or bone and are typically not accompanied by pain, but rather frequent episodes of bleeding. ${ }^{23}$ These lesions have a reliable natural history comprised of four distinct stages: quiescent, growing, symptomatic, and decompensating. ${ }^{24}$

Imaging plays an important role in the diagnosis of an AVM, but more so in operative planning. As with other vascular malformations, US and MRI can identify high flow patterns as well as determine the extent of the lesion. Lesions are often multispacial and hypervascular on color Doppler US. Magnetic resonance imaging is especially useful in defining 


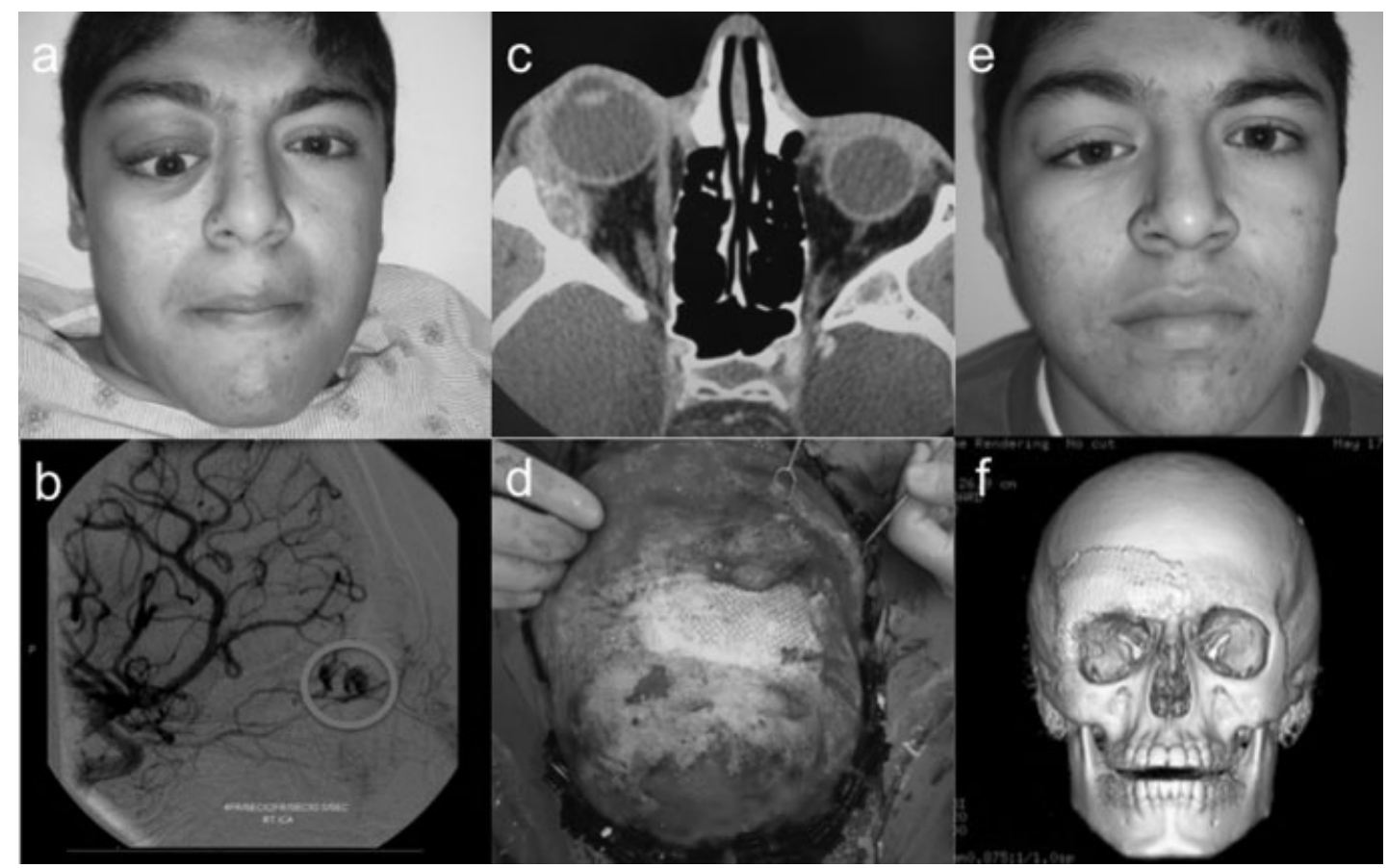

Fig. 8 A 12-year-old boy with biopsy-proven right periorbital arteriovenous malformation (AVM) causing (A) vertical dystopia and proptosis. (B) Angiogram was obtained confirming AVM, but was not amenable to embolization due to involvement of the ophthalmic artery. (C) Given the bony involvement, CT scan was useful for operative planning. (D) A radical resection was performed with subsequent orbital roof and forehead reconstruction with alloplast. (E,F) Vertical dystopia and proptosis was improved postoperatively.

the extent of AVMs, and typically shows numerous flow voids and hyperintense signal without an obvious mass. ${ }^{8}$ Unlike other vascular malformations, computerized tomography (CT) can be valuable, especially for bony AVMs. Angiography can also be utilized for defining the feeding and draining vessels prior to sclerotherapy or surgical intervention. ${ }^{5}$

Treatment of an AVM is based on the concept of obliteration of the nidus as this is thought to be responsible for the growth of the lesion through recruitment of new vessels from neighboring regions. Sclerotherapy and embolization remain first-line options to allow for safer intraoperative resection with less blood loss. ${ }^{25}$ Ligation of feeding vessels should never be done as this leads to rapid recruitment of collaterals and heightens vascularity. When considering resection of an AVM, it is paramount to realize that these lesions are rarely curable, but rather one should focus on disease control. Indications for intervention include ischemic pain, recurrent ulcerations/ bleeding, or perturbed cardiac function. ${ }^{26}$ Resection of these lesions can create large defects that may require flap coverage or formal reconstruction and should only be performed if the benefits greatly outweigh the risks ( - Fig. 8 ).

\section{Conclusion}

Vascular malformations are a source of great concern and anxiety not only for patients and their families, but also for the treating physicians. Proper identification as well as multidisciplinary approach is paramount for proper treatment. Understanding the clinical aspects, tools available for diagnosis, and options for interventions of each subtype of lesion will enable appropriate care to be provided and results to be maximized.

\section{References}

1 Mulliken JB, Glowacki J. Classification of pediatric vascular lesions. Plast Reconstr Surg 1982;70(1):120-121

2 Van Aalst JA, Bhuller A, Sadove AM. Pediatric vascular lesions. J Craniofac Surg 2003;14(4):566-583

3 Mulliken JB, Glowacki J. Hemangiomas and vascular malformations in infants and children: a classification based on endothelial characteristics. Plast Reconstr Surg 1982;69(3):412-422

4 Cohen MM Jr. Vascular update: morphogenesis, tumors, malformations, and molecular dimensions. Am J Med Genet A 2006; 140(19):2013-2038

5 Lowe LH, Marchant TC, Rivard DC, Scherbel AJ. Vascular malformations: classification and terminology the radiologist needs to know. Semin Roentgenol 2012;47(2):106-117

6 Colletti G, Valassina D, Bertossi D, Melchiorre F, Vercellio G, Brusati R. Contemporary management of vascular malformations. J Oral Maxillofac Surg 2014;72(3):510-528

7 Burrows PE, Mulliken JB, Fellows KE, Strand RD. Childhood hemangiomas and vascular malformations: angiographic differentiation. AJR Am J Roentgenol 1983;141(3):483-488

8 Tomlinson FH, Houser OW, Scheithauer BW, Sundt TM Jr, Okazaki H, Parisi JE. Angiographically occult vascular malformations: a correlative study of features on magnetic resonance imaging and histological examination. Neurosurgery 1994;34(5):792-799, discussion 799-800

9 Yuan KH, Gao JH, Huang Z. Adverse effects associated with photodynamic therapy (PDT) of port-wine stain (PWS) birthmarks. Photodiagn Photodyn Ther 2012;9(4):332-336

10 Buckmiller LM, Richter GT, Suen JY. Diagnosis and management of hemangiomas and vascular malformations of the head and neck. Oral Dis 2010;16(5):405-418 
11 Pappas DC Jr, Persky MS, Berenstein A. Evaluation and treatment of head and neck venous vascular malformations. Ear Nose Throat J 1998;77(11):914-916, 918-922

12 Hochman M, Adams DM, Reeves TD. Current knowledge and management of vascular anomalies, II: malformations. Arch Facial Plast Surg 2011;13(6):425-433

13 McCafferty IJ, Jones RG. Imaging and management of vascular malformations. Clin Radiol 2011;66(12):1208-1218

14 Dubois J, Garel L. Imaging and therapeutic approach of hemangiomas and vascular malformations in the pediatric age group. Pediatr Radiol 1999;29(12):879-893

15 Hein KD, Mulliken JB, Kozakewich HP, Upton J, Burrows PE. Venous malformations of skeletal muscle. Plast Reconstr Surg 2002; 110(7):1625-1635

16 Berenguer B, Burrows PE, Zurakowski D, Mulliken JB. Sclerotherapy of craniofacial venous malformations: complications and results. Plast Reconstr Surg 1999;104(1):1-11, discussion 12-15

17 Cabrera J, Cabrera J Jr, Garcia-Olmedo MA. Sclerosants in microfoam. A new approach in angiology. Int Angiol 2001;20(4):322-329

18 Eifert S, Villavicencio JL, Kao TC, Taute BM, Rich NM. Prevalence of deep venous anomalies in congenital vascular malformations of venous predominance. J Vasc Surg 2000;31(3):462-471
19 Burrows PE, Mitri RK, Alomari A, et al. Percutaneous sclerotherapy of lymphatic malformations with doxycycline. Lymphat Res Biol 2008;6(3-4):209-216

20 Perkins JA, Tempero RM, Hannibal MC, Manning SC. Clinical outcomes in lymphocytopenic lymphatic malformation patients. Lymphat Res Biol 2007;5(3):169-174

21 Adams MT, Saltzman B, Perkins JA. Head and neck lymphatic malformation treatment: a systematic review. Otolaryngol Head Neck Surg 2012;147(4):627-639

22 Stapf C, Mohr JP, Pile-Spellman J, Solomon RA, Sacco RL, Connolly ES Jr. Epidemiology and natural history of arteriovenous malformations. Neurosurg Focus 2001;11(5):e1

23 Christison-Lagay ER, Fishman SJ. Vascular anomalies. Surg Clin North Am 2006;86(2):393-425, x

24 Greene AK, Orbach DB. Management of arteriovenous malformations. Clin Plast Surg 2011;38(1):95-106

25 Lee BB, Do YS, Yakes W, Kim DI, Mattassi R, Hyon WS. Management of arteriovenous malformations: a multidisciplinary approach. J Vasc Surg 2004;39(3):590-600

26 Visser A, FitzJohn T, Tan ST. Surgical management of arteriovenous malformation. J Plast Reconstr Aesthet Surg 2011;64(3): 283-291 